\title{
MULTIRESOLUTION TERRAIN MODELING BASED ON TRIANGULATED IRREGULAR NETWORKS
}

\author{
HÉLIO PEDRINI
}

\begin{abstract}
RESUMO MODELAGEM EM MULTIRESOLUÇÃO DE TERRENOS BASEADA EM MALHAS TRIANGULARES Modelagem em multiresolução permite a representação, manipulação e visualização de grandes volumes de dados espaciais em múltiplos níveis de detalhe e precisão. Em sistemas de informação geográfica (SIG), áreas menos relevantes de um terreno podem ser descritas através de uma representação de menor resolução, enquanto alta resolução pode ser utlilizada apenas em áreas específicas de interesse. Este trabalho apresenta um método que permite a construção de uma sequiência de malhas triangulares através de operações de refinamento e simplificação, onde características relevantes dos terrenos são preservadas a cada etapa do processo de construção. Várias amostras de terrenos são utilizadas para demonstrar o desempenho do método proposto.
\end{abstract}

Palavras-chaves: Aproximação de superfícies, métodos de refinamento, simplificação de malhas, triangulação, múltiplos níveis de detalhe.

\begin{abstract}
Multiresolution modeling provides an abstraction for representing, manipulating, and visualizing large volumes of spatial data at multiple levels of detail and accuracy. In geographic information systems (GIS), a coarse representation can be used to describe less relevant areas of a terrain, while high resolution can be focused on specific parts of interest. This work presents a method for constructing a sequence of triangular meshes in the context of terrain modeling, where meshes are created through a set of simplification and refinement operations, while preserving relevant terrain features. The method has been tested on several terrain data sets.
\end{abstract}

Keywords: Surface approximation, refinement methods, mesh simplification, triangulation, multiple levels of detail.

INTRODUCTION The choice of a model to represent terrain data depends on several factors, such as the nature of the input data, application domain, and computational resources available. Digital models are frequently used to represent information of complex phenomena in order to allow a better understanding of their structure or behavior, and to provide a convenient way for investigation of the effects of inputs or changes to the model. Higher level of resolution or detail in the representation commonly require higher storage cost and computation time. Therefore, in order to be effective, a model should maintain a good trade-off between its resolution and storage requirements.

Although single, fixed resolution models are typically used to represent most current applications, it is often desirable that adaptive levels of detail can be dynamically extracted from the models. Multiresolution modeling offers the possibility of representing and analyzing terrains at variable resolution. Although multiresolution modeling is not a recent concept, there are few existing automatic tools for supporting variable resolution.

Heckbert \& Garland (1994) describe a number of data structures for multiresolution modeling, such as image pyramid, volume pyramid, and polygonal models. De Floriani \& Puppo (1989) propose a method based on a hierarchical subdivision described by a tree of nested triangulation. Gross et al. (1995), Bonneau (1998), and Lounsbery et al. (1997) propose techniques based on wavelets for constructing hierarchical representations of terrains.

The surface representation of a terrain can be generally viewed as a $2^{1 / 2}$-dimensional modeling problem, where a bivariate function $z=f(x, y)$ expresses the elevation $z$ of the surface at a point $(x, y)$ of the Euclidean plane. Therefore, any line parallel to $z$ axis penetrates the surface at most once. Although this representation covers most natural terrains, it excludes some features such as caves and faults.

A simple method for approximating terrain surfaces uses a regular grid digital elevation model (DEM), in which a set of sampled points representing measures of altitude or elevation are stored at regular intervals. A disadvantage of the DEM is its inherent spatial invariability, since the structure is not adaptive to the irregularity of the terrain. This may produce a large amount of data redundancy, especially where the topographic information is minimal. Alternatively, a triangulated irregular network (TIN) model approximates a topographic surface by a set of nonoverlapping contiguous triangular faces generated from a finite set of sampled data points. There are many advantages associated with irregular triangular networks. First, terrain data are commonly not regularly distributed in space. Second, terrain features can be incorporated into the model. For instance, vertices in a TIN can describe nodal terrain features such as peaks, pits or passes, while edges can represent linear terrain features such as break, ridge or channel lines. Third, TINs can be organized into a hierarchical model so that they can represent a terrain in various levels of detail.

Although a definite supremacy between both these models is difficult to establish due mainly to the wide variety of problems in terrain analysis and the computational resources available, TINs have become increasingly used because of their efficiency in storing irregularly spaced elevation data.

A multiresolution model based on polygonal surface representations using triangulated irregular meshes is described in this work. The algorithm uses Delaunay triangulation to construct the sequence of meshes, which reduces the occurrence of thin and long triangles since they can affect numerical stability and produce visual artifacts.

This paper initially reviews the main methods for creating an approximate terrain model. The proposed method is then described in detail and applied to real terrain data sets. Implementation issues and experimental results are presented and discussed. Finally, some final remarks are outlined.

RELATED WORK The main idea underlying a multiresolution terrain model (Chen \& Tobler 1986, De Berg \& Dobrindt 1998, Floriani et al. 1996, Voigtmann et al. 1987) is that a surface representation at an arbitrary level of detail can be extracted by either inserting more significant points into a coarse model or removing less significant points from a detailed model.

For many applications, piecewise-planar approximations of the terrain surface are considered sufficiently accurate, while allowing faster computation. On the other hand, functions of higher order can be used to obtain more precise and realistic representation of curved surfaces, however, requiring higher cost in computation time.

Most polygonal surface simplification methods found in the literature can be classified as refinement and decimation methods. Refinement methods start with a minimal initial approximation of the surface and repeatedly add new points to the triangulation until the model satisfies a specified approximation criterion. Decimation methods start with a triangulation containing the entire set of data points and iteratively simplify it, until the desired approximation criterion is achieved. 
Refinement Methods An intuitive refinement approach is to identify the most significant terrain characteristics and construct a triangulation based on these features. Topographic features typically include peaks, pits, passes, valleys and ridges.For instance, a peak is a point of relative maximum, while a point lies on a ridge if its neighborhood can be subdivided by a line passing through it. In this approach, the chosen points become the vertex set and the chosen lines become edges in a constrained triangulation.

The most commonly used technique for extracting topographic features from DEMs uses linear or nonlinear filters to examine elevation patterns in a neighborhood of points. Fowler \& Little (1979) suggest a two-step method for constructing TINs. The first step uses a $2 \times 2$ filter to identify topographic features, where an initial TIN is constructed using these points. In the second step, additional points are progressively added to the TIN until the model satisfies a specified error tolerance. Chen \& Guevara (1987) describe a method for selecting very important points (VIP) from DEMs, which evaluates the importance of a given point by calculating its contribution to the terrain surface. The importance of a point is based on the difference between its elevation and an estimated elevation from its eight neighbors. All points in the DEM are ordered in terms of importance. Point selection is based on either a predetermined significance level or a specified number of points.

Several criteria have been proposed to evaluate the approximation error, however, algorithms that use global error metrics are typically slow, while algorithms that generate lower quality or less compact approximations can generally be faster. (a)
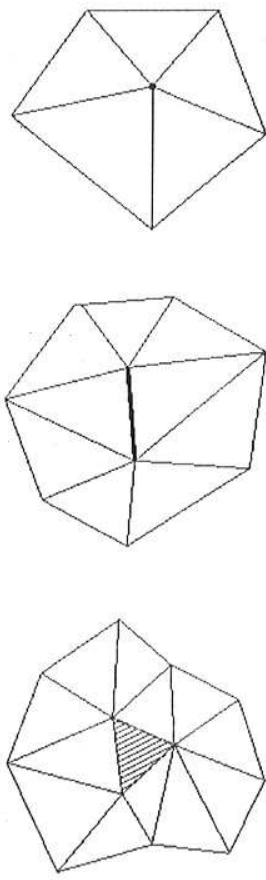
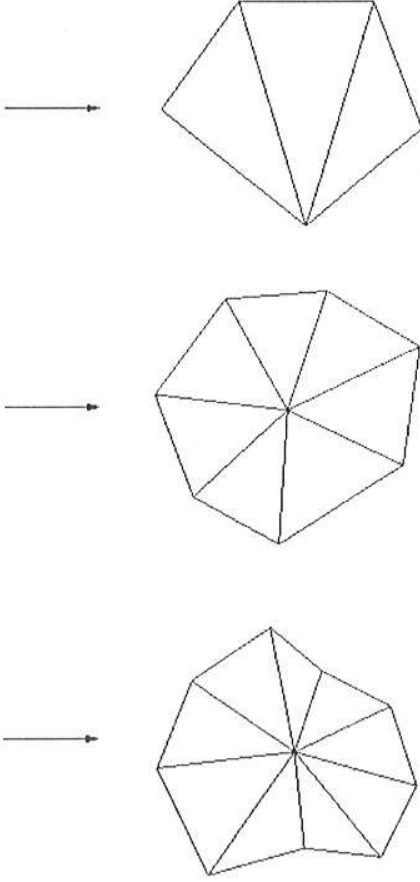

Figure I - (a) Vertex decimation; (b) edge decimation; (c) triangle decimation.

Decimation Methods Most decimation algorithms can be classified into three categories, according to the geometric entities (vertices, edges, or faces) used for removal. These categories are illustrated in Figure 1.

The fundamental goal of a decimation algorithm is to reduce the total number of triangles in a mesh, while preserving as accurately as possible important terrain features.

Lee (1989) proposes a drop heuristic method for simplifying terrains. The algorithm uses a vertex decimation approach, removing a vertex in each pass. An initial triangulation is created by adding an edge that connects two diagonal points in each $2 \times 2$ neighborhood. The error, measured as differences in elevation between surfaces, is computed for each remaining point in the triangulation, and the point with the lowest error is deleted.

Scarlatos \& Pavlidis (1990) present a method that uses curvature equalization to improve the triangulation. The size of the triangles with highest curvature is reduced by moving each vertex of the triangle. Unnecessary pairs of adjacent triangles are also removed by collapsing their common edge into a single point. Although their method can generally reduce the number of triangles, it can also increase the maximum error when explicit error tests are not used.

Schröder \& Roßbach (1994) describe a decimation algorithm in which the importance of a vertex is evaluated according to a measure of the roughness of the terrain at that vertex. A vertex is removed from the mesh if it does not affect the overall representation significantly. The area around the removed point is retriangulated.

A general vertex decimation algorithm is presented by Schroeder $e t$ al. (1992). The algorithm performs multiple passes over an existing triangulation, removing vertices until a specified error is achieved. The error at a vertex is calculated as the distance from the point to the average plane of the surrounding vertices.

Turk (1992) presents a method for simplifying polygonal surfaces by triangulating a new set of vertices which replaces the original one. An iterative point repulsion procedure is used to distribute the new set of points over the surface, concentrating more points in regions of higher curvature. The original points are then removed one by one, resulting a triangulation that preserves the topology of the original surface.

Hoppe et al. (1993) present a method for 3D surface simplification based on mesh optimization. Coarser triangulations are obtained from an initial fine triangulation by minimizing an energy function. The optimization process uses heuristics and random selection to either collapse, split, or swap an edge. Nonlinear optimization techniques are used to find the vertex positions that minimize the global error for a given topology. Topological changes are kept if they reduce the global error, otherwise they are discarded. A simplified version of this method is presented by Hoppe (1996), which uses a sequence of edge collapses to construct progressive meshes.

An edge-based decimation algorithm for simplifying surfaces is described by Guéziec \& Hummel (1995). To simplify the surface, edges are collapsed into a vertex, respecting certain geometrical and topological criteria. The simplification process minimizes a measure of error volume using linear programming. An edge collapse is considered valid if the topology of the surface is preserved, the normals of the modified faces change little, the new triangles are well shaped, and the error associated with the new vertex is within a threshold.

PROPOSED METHOD The central idea of our method is to produce a multiresolution representation such that, given an error tolerance, the extraction of an approximate model satisfying such tolerance can be obtained by means of a fast and compact procedure. The data structure needs to store only a mesh and a sorted sequence of vertices used to refine or simplify it.

The first step of our method is to generate a coarse piecewise linear approximation of the surface according to a predefined error tolerance. This initial triangulation is then refined by iteratively adding new points to the mesh, updating it after each point is inserted. An incremental algorithm is used to construct a constrained Delaunay triangulation, which offers the possibility of preserving relevant characteristics of the terrain such as peaks, pits, ridges, or valleys.

The vertex selection criterion is crucial during the triangulation process since it determines the degree of fidelity between the original data and the approximation. The most commonly used measure is based on the maximum difference between the actual elevation data and the surface approximation, known as $\mathrm{L}_{8}$ norm. This difference corresponds to a measure of local error. Another common error metric is the $L_{2}$ norm, which provides a measure of the average deviation between the original and the approximation. The $L_{2}$ norm produces a global error measure, however, the resulting algorithms are significantly slower than those using local metrics.

A variation to the conventional vertical error measure is proposed in this work as the vertex selection criterion, which is based on the 
maximum vertical error weighted by the standard deviation calculated in a $3 \times 3$ neighborhood of the candidate point (Pedrini 2000, Pedrini 2001). The idea is to associate greater importance to the points in regions where the local variability of the data is high, allowing the surface to conform to the local trends in the data. The vertices used to refine the mesh are stored in a priority queue. The corresponding approximation error is also assigned to each point in the list.

Conversely, a decimation algorithm is defined to produce a sequence of meshes through a set of vertex removal operations. At each iteration, the vertex with the smallest error is removed and the area affected by its removal is retriangulated. This process is repeated until a specified tolerance error is achieved.

The criterion for removing a vertex $v$ is computed by averaging the surface normals $n_{i}$ of the triangles surrounding $v$ weighted with their areas $A_{i}$ (see Figure 2) and taking the maximum angle, $\mathrm{a}_{m a r}$, between the averaged normal, $n_{u v}$, and the surrounding triangles, that is

$\alpha_{\max }=\max \left(\arccos \frac{\vec{n}_{a v} \cdot \vec{n}_{i}}{\left|\vec{n}_{a v}\right| \cdot\left|\vec{n}_{i}\right|}\right)$

where

$\vec{n}_{a v}=\frac{\sum \vec{n}_{i} \cdot A_{i}}{\sum A_{i}}$ and. $0 \leq \vec{n}_{i} \leq \pi$

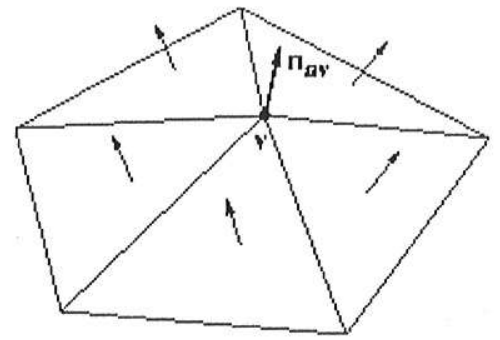

Figure 2 - Criterion for vertex removal.

Similarly to the refinement algorithm, each removed vertex is stored in a list as well as the corresponding maximum approximation error of the simplified mesh.

The sequence of local modifications generated during the refinement and simplification steps of a mesh can be viewed as a set of transformations

$\ldots M_{i-1} \Leftrightarrow M_{i} \Leftrightarrow M_{i+1} \cdots$

refinement $\rightarrow \leftarrow$ decimation

The extraction of a representation of the terrain at a given tolerance level is obtained by using a coarse triangulation and iteratively inserting vertices into the mesh until the desired precision is satisfied. If a given triangulation already guarantees a smaller error tolerance, then vertices are removed from the mesh, starting with the vertex with the smallest error.

RESULTS The proposed method was tested on a number of terrain data sets in order to evaluate the performance required to construct the multiresolution representation and extract different level of detail from it. Figure 3 illustrates the original digital elevation model of Lake Champlain (located between the states of New York and Vermont, USA) consisting of $1201 \times 1201$ points, where elevations range between $15 \mathrm{~m}$ and $1591 \mathrm{~m}$, standard deviation of $247 \mathrm{~m}$, and 3 - by 3 -arc-second data spacing ( 90 meters). The terrain model of Crater Lake (located in state of Oregon, USA), shown in Figure 4, contains 336x459 elevation points with range between $1533 \mathrm{~m}$ and $2478 \mathrm{~m}$, standard deviation of $162 \mathrm{~m}$, and $30-$ by $30-$ meter data spacing. These digital terrain models are derived from 1:250,000-scale and 1:25,000-scale maps, respectively.

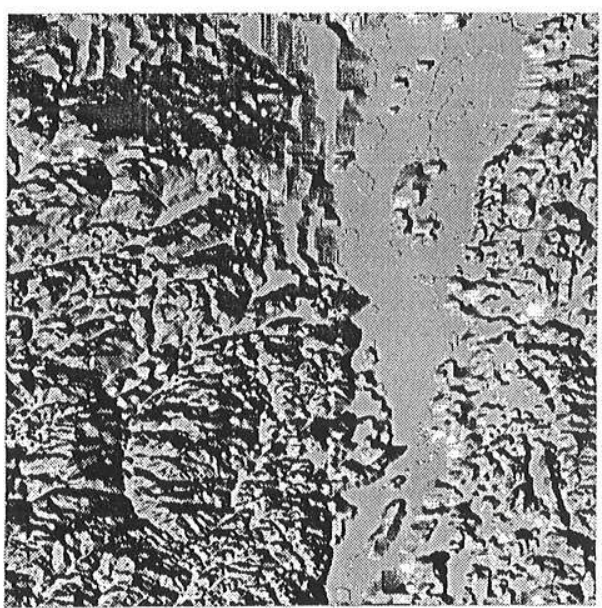

Figure 3 - The USGS Lake Champlain West DEM (I201x/20I points).

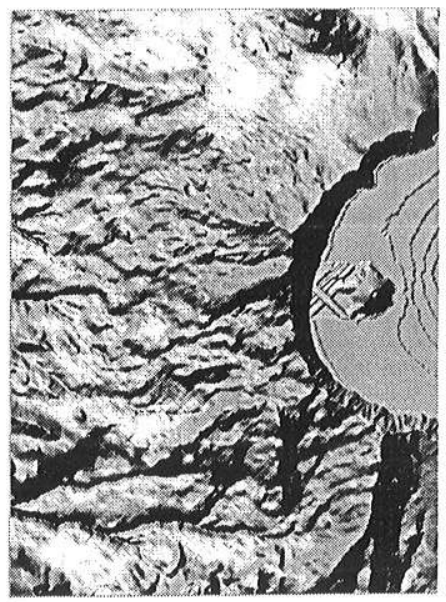

Figure 4 - The USGS Crater Lake West DEM (336x459 points)

Table I - Summary of results for USGS Lake Champlain West DEM (1201x/201).

\begin{tabular}{cc|c|cc}
$\begin{array}{c}\% \text { of } \\
\text { Points }\end{array}$ & $\begin{array}{c}\text { Number of } \\
\text { Triangles }\end{array}$ & $\begin{array}{c}\text { Local Error } \\
\text { (meters) }\end{array}$ & $\begin{array}{c}\text { RMS Error } \\
\text { (meters) }\end{array}$ & $\begin{array}{c}\text { CPU Time } \\
(\mathrm{sec})\end{array}$ \\
\hline 0.5 & 14,310 & 31.54 & 9.82 & 31.45 \\
\hline 1.0 & 28,554 & 23.00 & 6.53 & 35.25 \\
\hline 1.5 & 43,057 & 14.98 & 4.95 & 38.20 \\
\hline 2.0 & 57,228 & 12.97 & 3.60 & 42.31 \\
\hline 2.5 & 71,348 & 10.18 & 3.13 & 44.47 \\
3.0 & 85,926 & 9.03 & 2.55 & 48.62
\end{tabular}

Table 2 - Summary of results for USGS Crater Lake West DEM (336x459)

\begin{tabular}{ccccc}
$\begin{array}{c}\% \text { of } \\
\text { Points }\end{array}$ & $\begin{array}{c}\text { Number of } \\
\text { Triangles }\end{array}$ & $\begin{array}{c}\text { Local Error } \\
\text { (meters) }\end{array}$ & $\begin{array}{c}\text { RMS Error } \\
\text { (meters) }\end{array}$ & $\begin{array}{c}\text { CPU Time } \\
\text { (sec) }\end{array}$ \\
\hline 0.5 & 1,492 & 32.89 & 8.98 & 2.80 \\
\hline 1.0 & 3,006 & 24.25 & 6.76 & 3.07 \\
\hline 1.5 & 4,530 & 23.99 & 4.78 & 3.41 \\
\hline 2.0 & 6,063 & 23.44 & 3.99 & 3.70 \\
\hline 2.5 & 7,523 & 17.42 & 3.25 & 4.02 \\
\hline 3.0 & 9.120 & 10.15 & 2.93 & 4.30
\end{tabular}


Some results are summarized in Tables 1 and 2, where the percentage of selected points, number of triangles, precision of the mesh, and running time are indicated for six different levels. The running times are given in CPU seconds and the root mean square (RMS) error is calculated for each level. In the refinement step, the algorithm takes approximately 60 seconds to select 55,000 points on an SGI .O2 workstation (IRIX 6.5, R5000 with a $200 \mathrm{MHz}$ MIPS processor and 64 Mbytes of main memory).

Figures 5 and 6 show successive approximations for the digital elevation model of Lake Champlain and Crater Lake, respectively,
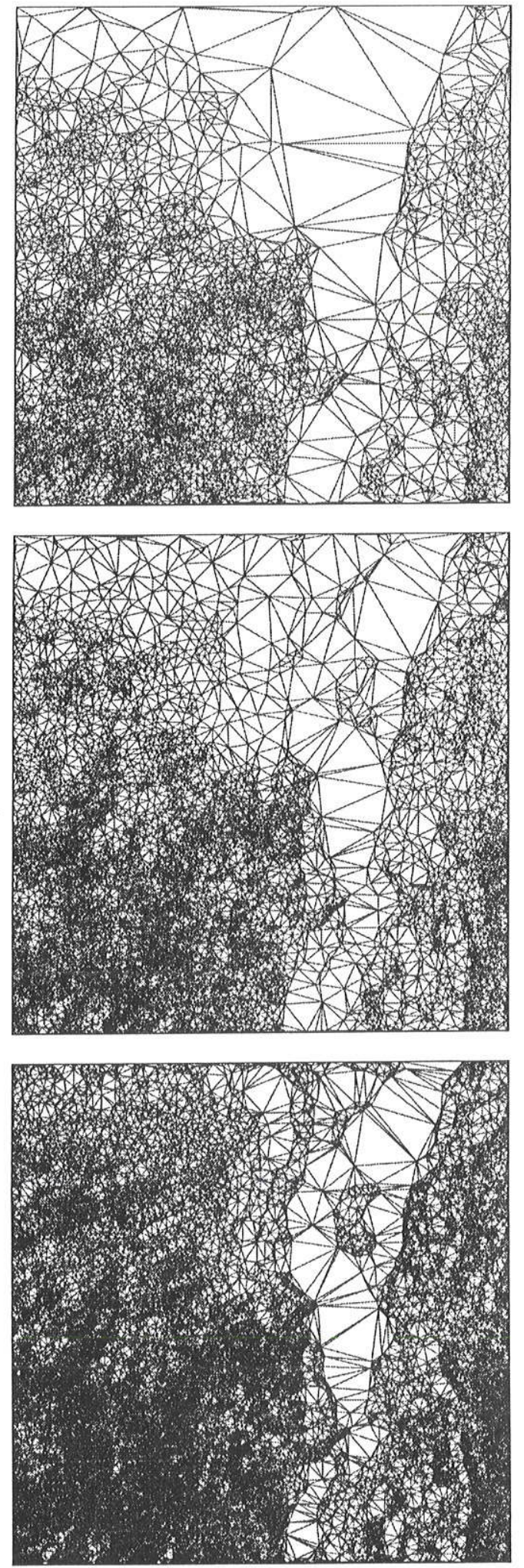

using $0.5 \%, 1.0 \%$, and $3.0 \%$ of the original points. Although these meshes use only a small percentage of the total number of points, they contain several features of the original terrain.

Figures 7 and 8 show the RMS error and the new local vertical error for Lake Champlain and Crater Lake, respectively. Even though the local error metric does not guarantee that the triangular surface at a given step is more accurate than the one calculated at the previous step, its behavior is decreasing, although not monotonically.

The vertical error of the approximate surface of the Lake Champlain using $5 \%$ of the input points is shown in Figure 9(a), which
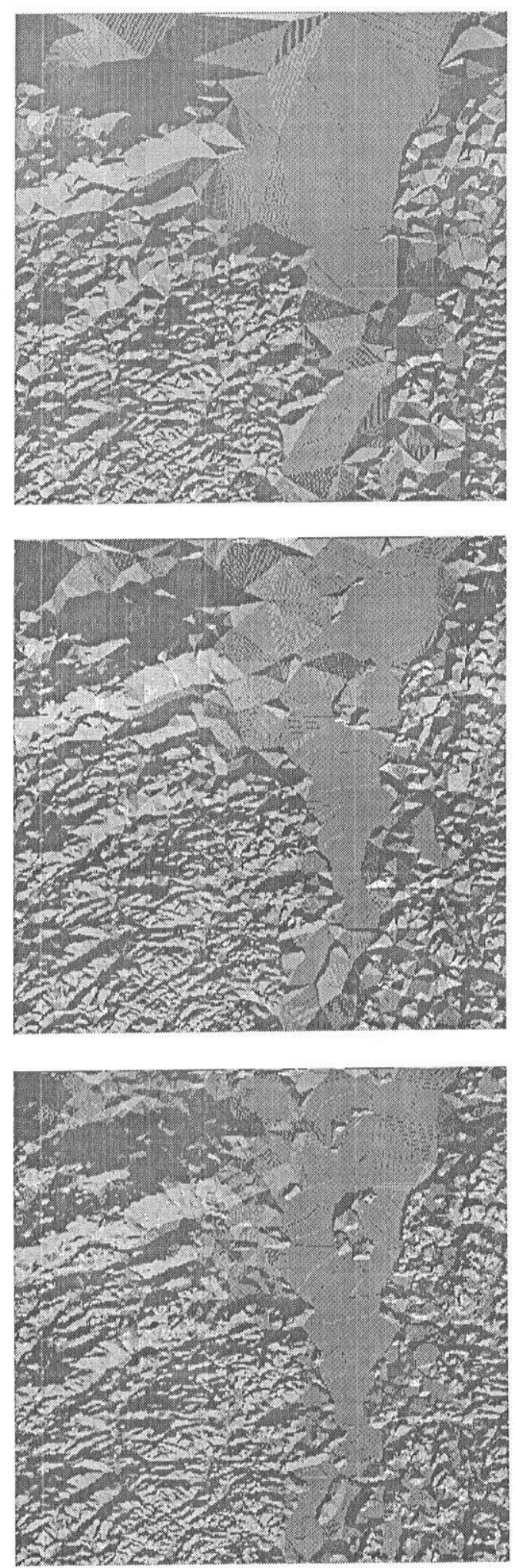

Figure 5 - Three approximations of the Lake Champlain DEM using 0.5\%, 1.0\%, and 3.0\% of the original points, respectively. 

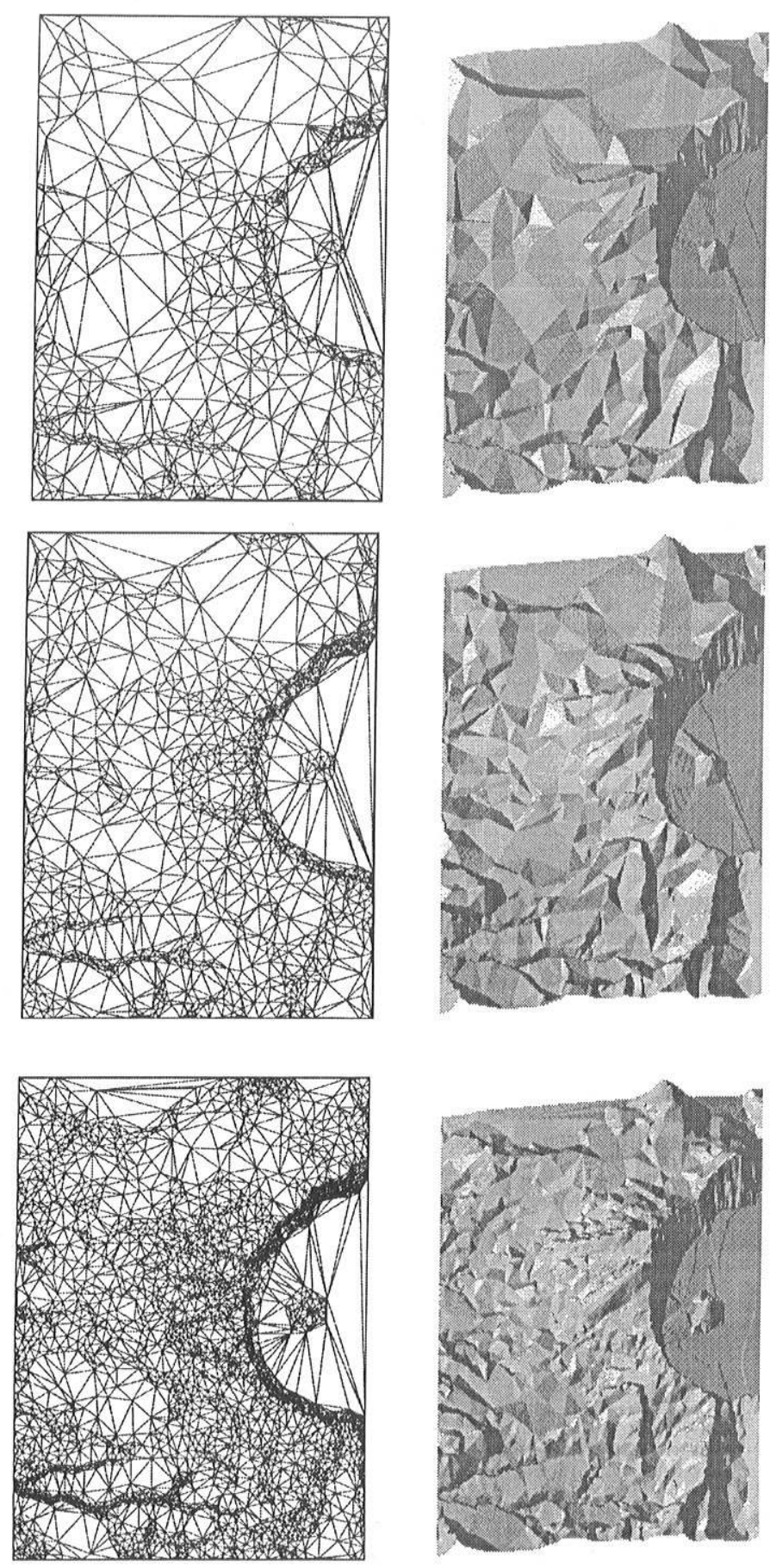

Figure 6 - Three approximations of the Crater Lake DEM using 0.5\%, 1.0\%, and $3.0 \%$ of the original points, respectively.

is quite flat. Figures 9(b) and 9(c) show the error profile in horizontal (parallel to the $x$-axis) and vertical direction (parallel to the $y$-axis), respectively. 1-D cross-sections of elevations in both directions are given in Figures 10(a) and 10(b), where the solid and dotted lines represent the original and the approximate elevation values, respectively. The vertex with maximum vertical error is used as the intersection point of the two cross-sections. Note that there is no significant deviation between the original and the approximate surfaces.

The results have demonstrated a good balance between speed and ability to process large terrain data sets, producing high quality approximations with great flexibility.

CONCLUSIONS The method described in this work extracts terrain representations at different levels of detail through a set of

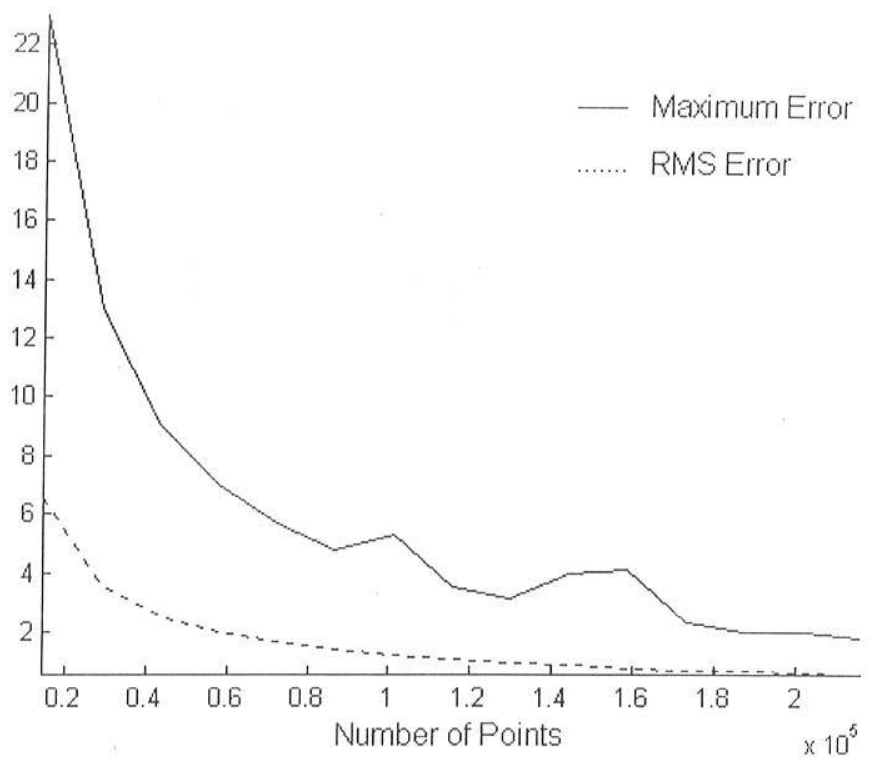

Figure 7 - Approximation error for Lake Champlain.

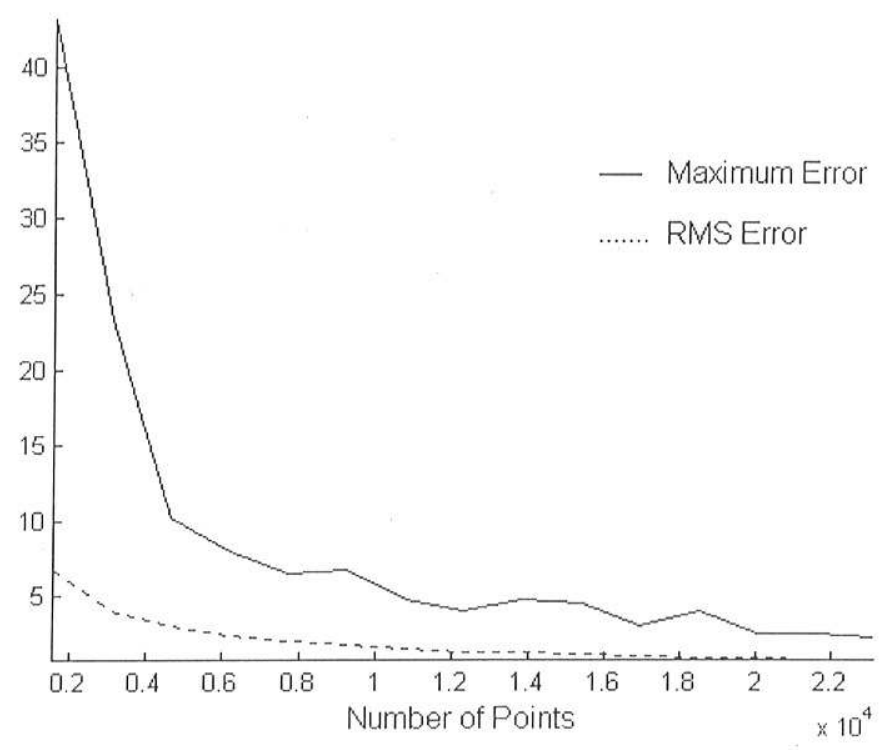

Figure 8 - Approximation error for Crater Lake.

simplification and refinement operations. While many multiresolution modeling techniques found in the literature (De Floriani 1989, De Floriani \& Puppo 1995) are based on hierarchical subdivision, our method performs a linear sequence of local updates on an initial mesh. This process provides a flexible and compact model for representing terrain surfaces maintaining a good trade-off between computational efficiency and space requirements.

The proposed algorithm uses an iterative refinement process to increase the level of details and a vertex decimation to simplify models. A new local error metric is used to select points to be inserted into the mesh, while a measure of angle between surface normals is used to determine whether a vertex should be removed from the mesh.

It is expected that higher quality representations can be obtained by constructing a curved approximation of the original mesh through higher-order approximating surfaces, instead of piecewise-planar subdivisions. However, additional work is need to establish practical merits of such techniques in the context of terrain modeling.

Acknowledgements This paper was partially supported by a grant from Conselho Nacional de Desenvolvimento Científico e Tecnológico, CNPq, Brazil. The digital elevation models are courtesy of the United States Geological Survey. To two anonymous referees of RBG for the suggestions to the originals. 

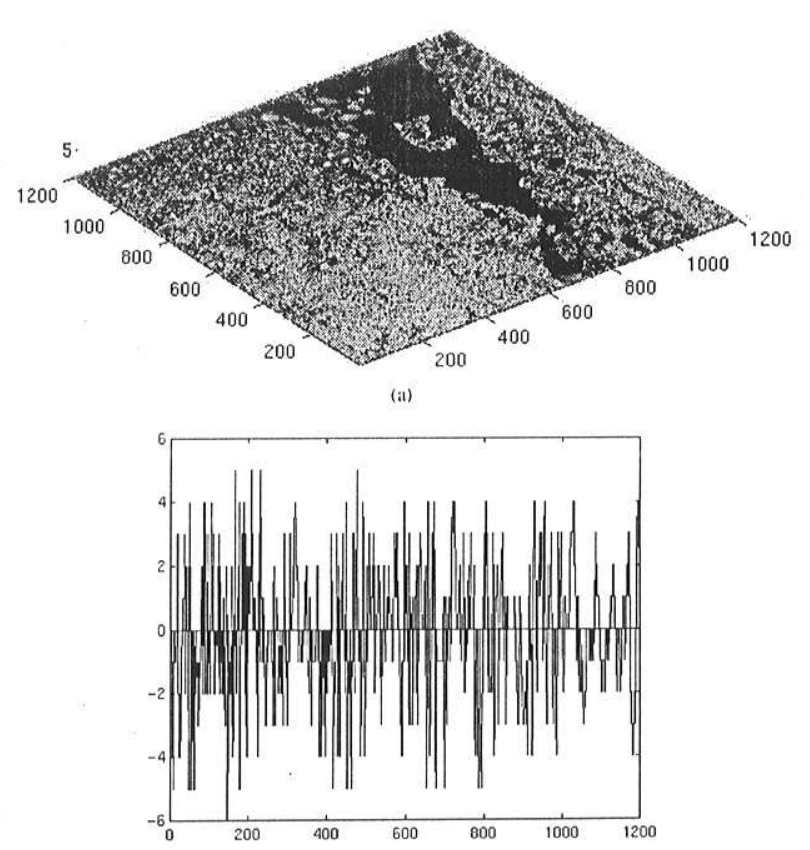

(b)

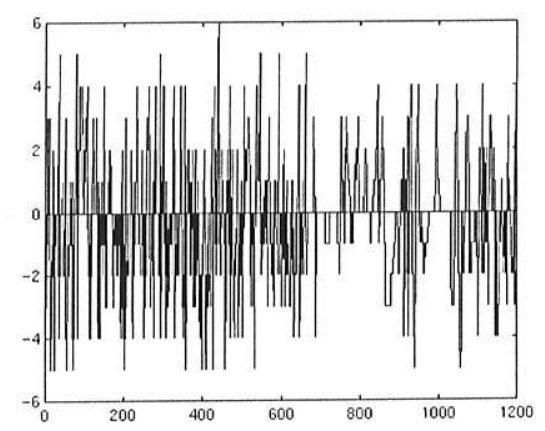

(c)

Figure 9 - (a) Three-dimensional plot of the elevation error for Lake Champlain (using 5\% of the input points); (b) error profile at 26th row; (c) error profile at 150th column.

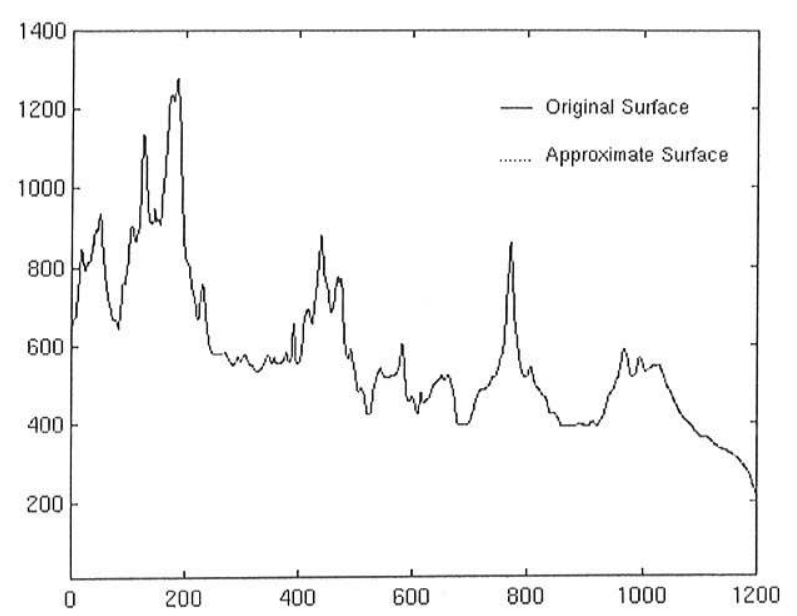

(i)

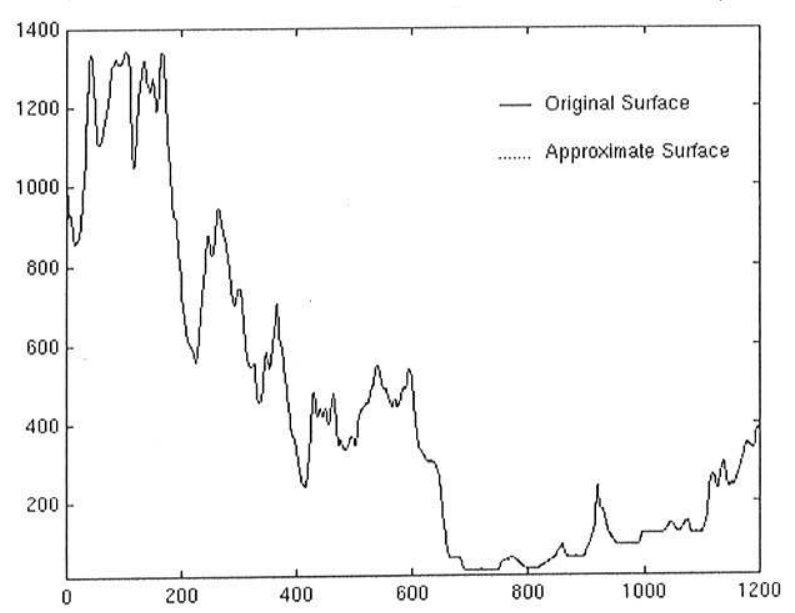

(b)

Figure 10 - (a) I-D cross section of original and approximate surfaces of the Lake Champlain at 26th row; (b) $1-D$ cross section of original and approximate surfaces at 150th column.

\section{References}

Bonneau G.P 1998. Multiresolution analysis on irregular surface meshes. IEEE Transactions on Visualization and Computer Graphics, 4:365-378.

Chen Z \& Guevara J.A. 1987. Systematic selection of very important points (VIP) from digital terrain model for constructing triangular irregular networks. In: Eighth International Symposium on Computer-Assisted Cartography - Auto-Carto 8 Baltimore, Maryland, USA, Proceedings, p. 50-56.

Chen Z.T. \& Tobler W.R. 1986. Quadtree representation of digital terrain, In: Auto-Carto, London, England, Proceeding.s, p. 475-484.

De Berg M. \& Dobrindt K.T.G. 1998. On levels of detail in terrains, Graphical Models and Image Processing, 60:1-12.

De Floriani L. 1989. A pyramidal data structure for triangle-based surface description. IEEE Computer Graphics and Applications, 9:67-78.

De Floriani L., Marzano P., Puppo E. 1996. Multiresolution models for topographic surface description, The Visual Computer, 12:317-345.

De Floriani L. \& Puppo E. 1995. Hierarchical triangulation for multiresolution surface description. ACM Transactions on Graphics, 14:363-411.

Fowler R.J. \& Little J.J. 1979. Automatic extraction of irregular network digital terrain models. Computer Graphics, 13:199-207.

Gross M.H. Gatti R., Staadt O. 1995. Fast multiresolution surface meshing. In: IEEE Visualization'95, Atlanta, Georgia, USA, Proceedings, p. 135-142.

Guéziec A. \& Hummel R. 1995. Exploiting triangulated surface extraction using tetrahedral decomposition. IEEE Transactions on Visualization and Computer Graphics, 1:328 342.

Heckbert P.S. \& Garland M. 1994. Multiresolution modeling for fast rendering. In: Graphics Interface'94, Banff, Alberta, Canada, Proceedings, p. 43-50.

Hoppe H. DeRose T. Duchamp T., McDonald J., Stuetzle W. 1993. Mesh optimization. In: SIGGRAPH'93 Conference, Anaheim, California, USA, Proceedings, p. 19-26.
Hoppe H. 1996. Progressive meshes. In: SIGGRAPH'96 Conference, New Orleans, Louisiana, USA, Proceedings, p. 99-108.

Lee J. 1989. A drop heuristic conversion method for extracting irregular network for digital elevation models. In: GIS/LIS'89, American Congress on Surveying and Mapping, Proceedings, Vol.. 1, p. 30-39.

Lounsbery M., Derose T., Warren J. 1997. Multiresolution analysis for surfaces of arbitrary type. ACM Transactions of Graphics, 16:34-73.

Pedrini H. 2000. An Adaptive Method for Terrain Surface Approximation based on Triangular Meshes. Rensselaer Polytechnic Institute, Troy, NY, USA, PhD Thesis, 46 p.

Pedrini H. 2001. An improved refinement and decimation method for adaptive terrain surface approximation. In: 9th International Conference in Central Europe on Computer Graphics, Visualization and Computer Vision, Plzen, Czech Republic, Proceedings, p. 103-109.

Scarlatos L. L. \& Pavlidis T. 1990. Hierarchical triangulation using terrain features. IEEE Visualization'90, San Francisco, California, USA, Proceedings, p. 168-175.

Schröder F. \& Robbach P. 1994. Managing the complexity of digital terrain models. Computer \& Graphics, 18:775-783.

Schroeder W. J., Zarge J. A., Lorensen W. E. 1992. Decimation of triangle meshes. Computer Graphics, 26:65-70

Turk G. 1992. Re-tiling polygonal surfaces. Computer Graphics, 26:55-64.

Voigtmann A., Becker L., Hinrichs K. 1997. A hierarchical model for multiresolution surface reconstruction. Graphical Models and Image Processing, 59:333-348.

Manuscrito A-1132

Recebido em 01 de novembro de 1999 Revisão do autor em 12 de abril de 2001 Revisão aceita $\mathrm{em} \mathrm{em} 17$ de abril de 2001 